\title{
Acupoint Application in Patients with Chronic Stable Angina Pectoris: Study Protocol of a Randomized, Double-Blind, Controlled Trial
}

\author{
Yulan Ren, ${ }_{1}^{1}$ Dehua Li, ${ }^{2}$ Hui Zheng, ${ }^{1}$ Junling Lv, ${ }^{1}$ Junyan Leng, ${ }^{1}$ Linglin Zhang, \\ Jie Zhang, ${ }^{1}$ Hailong Fan, ${ }^{1}$ and Fanrong Liang ${ }^{1}$ \\ ${ }^{1}$ The Department of Acupuncture \& Tuina, Chengdu University of Traditional Chinese Medicine, Chengdu, Sichuan 610075, China \\ ${ }^{2}$ The Department of Acupuncture, Teaching Hospital of Chengdu University of Traditional Chinese Medicine, Chengdu, \\ Sichuan 610072, China
}

Correspondence should be addressed to Fanrong Liang; acuresearch@126.com

Received 24 March 2014; Revised 25 June 2014; Accepted 26 June 2014; Published 27 August 2014

Academic Editor: Karl Wah-Keung Tsim

Copyright (C) 2014 Yulan Ren et al. This is an open access article distributed under the Creative Commons Attribution License, which permits unrestricted use, distribution, and reproduction in any medium, provided the original work is properly cited.

\begin{abstract}
Background. Chronic stable angina pectoris (CSAP) is a major syndrome of ischemic heart disease (IHD). CSAP manifests as chest pain or discomfort and affects patients' quality of life. Acupoint application (AP) has been reported to be effective for managing the symptoms of CSAP, but the evidence is not convincing. Therefore, we designed a randomized, double-blind, placebo-controlled clinical trial to evaluate the efficacy of AP in the treatment of CSAP. Methods and Analysis. Two hundred participants with CSAP will be randomly assigned in a 1:1:1:1 ratio into 4 groups. All participants will receive 12 sessions of treatment in 4 weeks and the same basic treatment procedure. The participants will be visited and assessed for 12 weeks, including a 4-week screening, a 4-week treatment phase, and a 4-week follow-up phase. The primary outcome is the change in the total frequency of self-reported angina attack at 4th week compared with the baseline. The secondary outcomes include the intensity of angina pain, consumption of nitroglycerin or Suxiao Jiuxin pills, CCS angina classification, SAQ, SAS and SDS score. Ethics. The study protocol has been reviewed and approved by the Sichuan Regional Ethics Review Committee on TCM (number 2013kl-001). This trial is registered with clinicaltrials.gov NCT02029118.
\end{abstract}

\section{Introduction}

Ischemic heart disease (IHD) is the leading cause of death in high-income and in low- and middle-income countries and accounted for $17.3 \%$ and $11.8 \%$ of total deaths, respectively [1]. Chronic stable angina pectoris (CSAP) is the most prevalent manifestation of IHD and affects up to $5 \%$ of the over-40-year-old population in most developed countries. In every one million people in the general population of most European countries, it is estimated that 20,000 to 40,000 individuals suffer from CSAP $[2,3]$.

CSAP patients complain of severe chest pain or discomfort in the chest or adjacent areas. The pain and discomfort are caused by severe atherosclerotic narrowing of one or more coronary arteries. The current standard of care for CSAP relies on strategies that not only relieve symptoms and prolong angina-free walking but also reduce the incidence of adverse clinical outcomes. These strategies include lifestyle changes, aggressive management of modifiable coronary artery disease risk factors, pharmacological therapy, and myocardial revascularization $[4,5]$. Despite improved diagnostic techniques, medical therapy, and revascularization procedures, many patients continue to suffer from angina $[2,5]$. However, widely used antianginal therapies have side effects, including headache, postural hypotension and dizziness, and the continuous uptake of nitrates may lead to drug tolerance [6]. Therefore, complementary therapies are popular among CSAP patients [7,8].

As one of the complementary therapies, acupoint application (AP) is an external therapy used in traditional Chinese medicine (TCM); with the aim of preventing and treating diseases, herbal medicines are made into plaster 


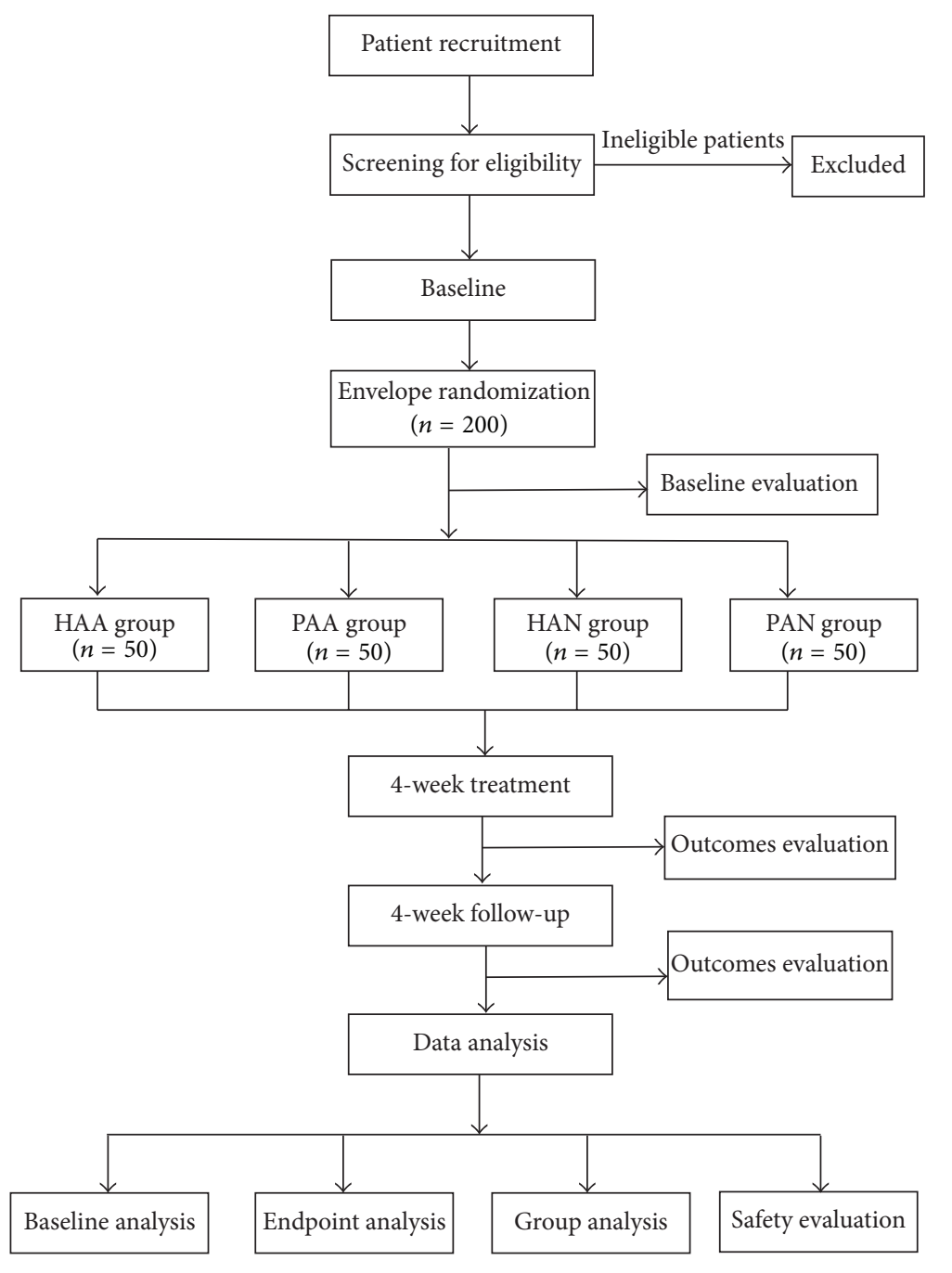

Figure 1: Study design.

to be applied on certain acupoints. The treatment relies on the mechanism of percutaneous absorption, which is currently under study internationally for transdermal drug delivery [9]. AP is a straightforward procedure with few side effects. AP has a long history in China and is widely used as an alternative and complementary therapy for the treatment of stable angina. Prospective clinical studies have confirmed that AP may alleviate symptoms and improve heart function in patients with stable angina [10-12]. Experimental research has revealed that AP may alter electrical and mechanical cardiac activities, strengthen myocardial substrate metabolism in the margin of the ischemic region, and minimize myocardial ischemic injury [13]. However, the majority of published studies have been small-sample observational studies rather than intervention studies. A lack of high-quality randomized, controlled, trial results means that there is insufficient evidence for the use of AP as a therapy for stable angina. To provide more persuasive evidence, we designed a randomized, controlled, double-blind clinical trial to evaluate the efficacy and safety of AP in patients with CSAP.

\section{Methods}

2.1. Design. This study is a randomized, double-blind, placebo-controlled trial that was designed to evaluate the efficacy and safety of AP in patients with CSAP in China. A total of 200 participants will be randomized (in a $1: 1: 1: 1$ ratio) into four groups: a herbal medicine application on acupoints (HAA) group, placebo application on acupoints (PAA) group, herbal medicine application on nonacupoints (HAN) group, and placebo application on nonacupoints (PAN) group (Figure 1). Eligible patients will be recruited from the Chengdu Chronic Disease Hospital, General Hospital of the Chengdu Military Region of the People's Liberation Army, and Third Affiliated Hospital of Chengdu University of Traditional Chinese Medicine.

The study protocol was approved by the Sichuan Regional Ethics Review Committee on TCM (number 2013kl-001) in January 2013. The study protocol follows the principles of the CONSORT and STRICTA statements as well as the Declaration of Helsinki (Sixth revision, 2008). Each patient 
will sign an informed consent form prior to any study procedure being conducted. Meanwhile, the patients will have sufficient time to decide whether they will participate in this study or select other treatments.

2.2. Study Flow. When a patient with CSAP visits a doctor in the cardiovascular department of one of the three study centers, the doctor will provide information about the trial and ask if the patient would like to participate in the study.

First, the patient will be informed. After enrollment, the patient will receive basic treatment combined with AP with herbal medicine/placebo plaster on acupoints/nonacupoints.

During the observation period of 12 weeks, patients are not permitted to receive any other treatments except drugs for immediate relief of acute symptoms of CSAP.

If a patient consents, the investigator will ask questions to satisfy the inclusion and exclusion criteria. The investigator will also collect demographic data and information about medical history, concomitant medication, physical examination, vital signs, and regular test results. If the patient is eligible, he or she will be randomly assigned to one of the four groups based on a sequence sealed in an opaque envelope. The following parameters will be evaluated at baseline: the frequency of angina attack, consumption of nitroglycerin or Suxiao Jiuxin pills, visual analogue scale (VAS) score, Canadian Cardiovascular Society (CCS) angina classification, Seattle Angina Questionnaire (SAQ) score, self-rating anxiety scale (SAS) score, and self-rating depression scale (SDS) score. Efficacy and safety will be evaluated at the 4 th week and 8 th week.

Each patient will be required to complete a participant diary to record symptoms, medications, and adverse events (AEs); the diaries will be given to the investigator at every visit.

2.3. Randomization and Blinding. Complete randomization will be stratified for centers in this study. Using the method of complete randomization, the randomization codes will be generated by Statistical Analysis Software (SAS, 9.1). Randomization codes will be managed by a statistician who will not be involved in the study. The plaster will be prepared beforehand by the central pharmacy and will be marked with labels and randomization codes. To ensure that the assigned intervention is concealed, the investigators will obtain an opaque, sealed envelope containing each participant's assigned intervention from the statistician just before the procedure is performed. The sealed envelope will be opened when an eligible patient is enrolled in the study. Then, the participant will be treated according to the allocation specified in the envelope. The investigators, participants, efficacy evaluators, and statisticians will all be blinded to the intervention allocations.

\subsection{Participants}

2.4.1. Inclusion Criteria. Patients will be diagnosed according to the CSAP criteria that are issued by the American College of Cardiology/American Heart Association (ACC/AHA). Eligible participants are male or female, are aged between 35 and 85 years, have at least a 3-month history of stable angina, have at least 2 angina episodes per week, and agree to provide written informed consent.

2.4.2. Exclusion Criteria. Patients will be excluded if they have a history of diabetes, coinfection, blood disorders, allergies, or participation in other clinical trials. Pregnant and/or lactating women will also be excluded.

2.4.3. Participant Recruitment. The cardiologists will locate patients with CSAP and suggest that they participate in the study. The investigator will screen eligible patients according to the inclusion/exclusion criteria. Potential patients in local communities will be informed of the study through advertisements. Patients will be enrolled only if they meet the inclusion criteria and provide written informed consent.

2.5. Interventions. To ensure participant safety, we will comply with the European and Chinese guidelines for the management of patients with CSAP $[5,14]$. All participants in the four groups will undergo the same basic treatment procedure. Additionally, following the guidelines, short-acting sublingual nitroglycerin therapy will be prescribed to all patients for the immediate relief of acute symptoms.

2.5.1. Basic Treatment. Basic treatment includes health education and standard medications. Health education includes recommendations for lifestyle changes, such as increased exercise, reduced alcohol consumption, weight loss, and smoking cessation. Standard medications include $100 \mathrm{mg} / \mathrm{d}$ aspirin QD, $25 \mathrm{mg} / \mathrm{d}$ metoprolol BID, $5 \mathrm{mg} / \mathrm{d}$ ramipril QD, and $20 \mathrm{mg} / \mathrm{d}$ atorvastatin QN $[5,14]$.

2.5.2. Plaster Production. In this study, the plasters for the AP therapy are divided into herbal medicine and placebo plasters. The herbal medicine plaster originates from a traditional Chinese decoction known as "guanxin suhe wan," which was recorded in "The formulae of the Peaceful Benevolent Dispensary in the Song Dynasty." This recipe includes styrax, borneol, frankincense, sandalwood, trichosanthes kirilowii Maxim, allium macrostemon Bunge, leeches, corydalis yanhusuo, and other ingredients. Clinical studies and animal experiments have identified a protective effect from this decoction on myocardial ischemia and hypoxia [15-19]. For the herbal medicine plaster, herbal medicine will be ground into fine powder and then mixed with honey to form a paste, which will be adhered to a special $5 \times 5 \mathrm{~cm}$ sticking plaster with a $1.6 \mathrm{~cm}$-diameter container in the middle. The placebo plaster will be made of buckwheat flour without the herbal medicines, but with the addition of honey and flavoring agents, the placebo plaster will have the same appearance, color, and smell as the herbal medicine plaster.

2.5.3. Acupoint Selection. The acupoint selection regimen was developed by acupuncturists and cardiovascular specialists based on traditional acupuncture theories and our earlier literature review, which revealed that acupoints on the pericardium meridian were chiefly selected for angina 


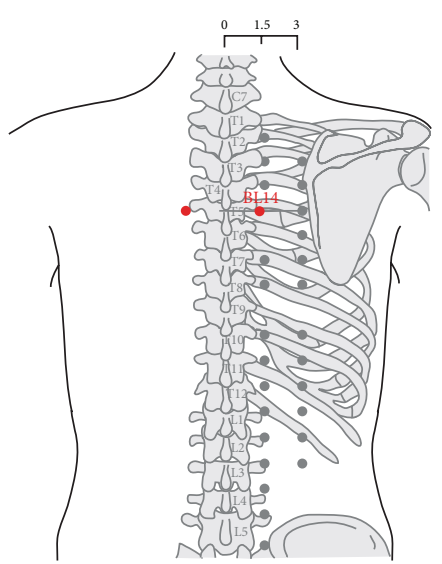

Jueyinshu (BL14)

Location: in the upper back region, at the same level as the inferior border of the spinous process of the fourth thoracic vertebra (T4), 1.5 B-cun lateral to the posterior median line.

From: WHO standard acupuncture point locations in the Western Pacific region

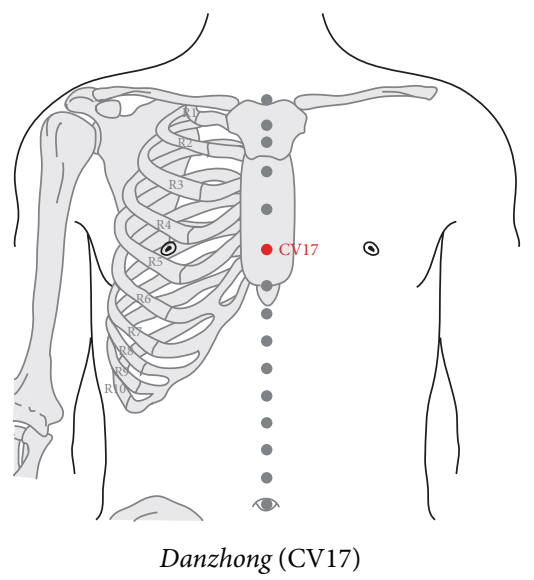

Location: in the anterior thoracic region, at the same level as the forth intercrostal space, on the anterior median line.

From: WHO standard acupuncture point locations in the Western Pacific region

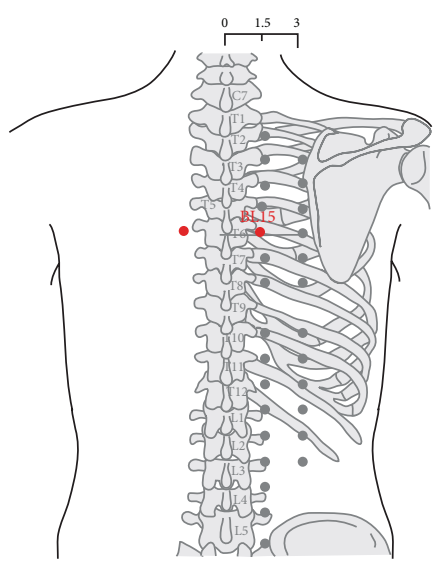

Xinshu (BL15)

Location: in the upper back region, at the same level as the inferior border of the spinous process of the fifth thoracic vertebra (T5), 1.5 B-cun lateral to the posterior median line.

From: WHO standard acupuncture point locations in the Western Pacific region

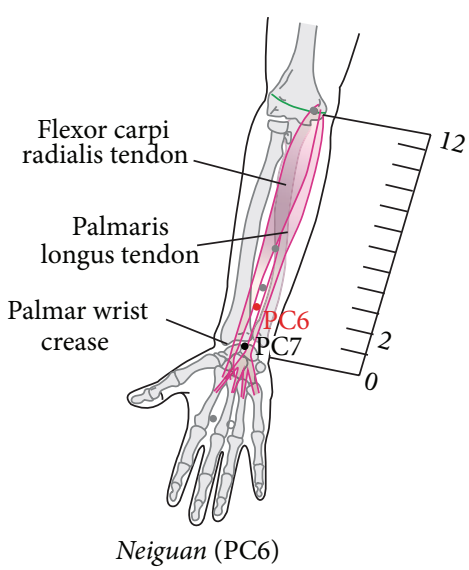

Location: on the anterior aspect of the forearm, between the tendons of the palmaris longus and the flexor carpi radialis, $2 \mathrm{~B}$-cun proximal to the palmar wrist crease. From: WHO standard acupuncture point locations in the Western Pacific region

FIgURE 2: Diagram of acupoints location.

[20]. The following acupoints will be used: Jueyinshu (BL14), Xinshu (BL15), Danzhong (RN17), and Neiguan (PC6); the names/codes and locations of all acupoints comply with WHO standards [21] (Figure 2). The nonacupoints are located in the same spinal segment but $2 \mathrm{~cm}$ away from the acupoints and outside the Chinese meridian system [22]. All acupoints and nonacupoints will be bilaterally used.

2.5.4. Study Group. All groups will undergo the same basic treatment procedure, which continues from the baseline to the end of the follow up.
From the beginning of the study to the end of 4 th week, the participants in the HAA group will receive the treatment of herbal medicine plaster on the acupoints and the participants in the PAA group will receive the treatment of placebo plaster on the acupoints; similarly, the participants in the HAN group will receive the treatment of herbal medicine plaster on the nonacupoints and the participants in the PAN group will receive the treatment of placebo plaster on the nonacupoints (Table 1). The operating procedure is to locate the acupoints, disinfect the skin, and attach the plaster on the acupoints. A total of 12 treatments will be performed during 
TABLE 1: Details of study groups.

\begin{tabular}{|c|c|c|}
\hline Group & Acupoints & Interventions \\
\hline $\begin{array}{l}\text { HAA } \\
\text { group }\end{array}$ & \multirow{2}{*}{$\begin{array}{l}\text { Jueyinshu (BL14), Xinyu (BL15) } \\
\text { Danzhong (CV17), and Neiguan } \\
\text { (PC6) }\end{array}$} & $\begin{array}{l}\text { Herbal medicine } \\
\text { application, acupoint, } \\
\text { and basic treatment }\end{array}$ \\
\hline $\begin{array}{l}\text { PAA } \\
\text { group }\end{array}$ & & $\begin{array}{l}\text { Placebo application, } \\
\text { acupoint, and basic } \\
\text { treatment }\end{array}$ \\
\hline $\begin{array}{l}\text { HAN } \\
\text { group }\end{array}$ & \multirow{2}{*}{$\begin{array}{l}\text { Nonacupoints in the same spinal } \\
\text { segment as those in the } \\
\text { acupoints of HAA group but } \\
2 \mathrm{~cm} \text { away from genuine } \\
\text { acupoints and outside the } \\
\text { Chinese meridian system }\end{array}$} & $\begin{array}{l}\text { Herbal medicine } \\
\text { application, } \\
\text { nonacupoint, and } \\
\text { basic treatment }\end{array}$ \\
\hline $\begin{array}{l}\text { PAN } \\
\text { group }\end{array}$ & & $\begin{array}{l}\text { Placebo application, } \\
\text { nonacupoint, and } \\
\text { basic treatment }\end{array}$ \\
\hline
\end{tabular}

the 4 weeks, with one 6-8-hour treatment every other day for 6 days and one day of rest per week.

The plasters should be removed in advance if the local skin develops an allergic reaction to the medicine, which manifests as pruritus, redness, and erythra. When acute angina attack happens, the participant will receive an antianginal drug (nitroglycerin, nifedipine, or Suxiao Jiuxin pills [23]) according to the participant's previous treatment history and individual contraindications. For the majority of the participants, we will recommend short-acting sublingual nitroglycerin. Regardless of the medications, participants will be instructed to record drug information, including name, administration time, and dosage. The antianginal drugs will be provided for free. Nitroglycerin (Beijing Yimin Pharmaceutical Co., Ltd., Beijing, China), which is approved by the China Food and Drug Administration (CFDA, number H11021022), will be provided as a sublingual dose of $0.5 \mathrm{mg}$ (one tablet); nifedipine tablets (CSPC Pharmaceutical Group Limited, Shijiazhuang, China, CFDA H13021315) will be provided as an oral dose of $10 \mathrm{mg}$ (one tablet); finally, Suxiao Jiuxin pills (SX) (Zhongxin Pharma Tianjin number 6 Traditional Chinese Medicine Factory, Tianjin, China, CFDA Z12020025) will be provided as a sublingual dose of 5-10 pills. Other antianginal drugs will be prohibited. In the case of taking prohibited drugs, participants will be eliminated from the analysis due to protocol violation.

2.6. Investigator Training and Quality Control. All investigators involved in this study will receive theoretical and practical training courses to ensure that the operation is completely standardized. The training will include how to screen the eligible participants, use the random envelope, instruct participants to complete the diary cards, complete the case report form and assess efficacy and safety, and select acupoints and apply the plaster. After the training, all investigators will take a test. If they pass the test, they will obtain a certificate.

To guarantee the quality of the study, quality control is carried out every 3 months; that is, an inspection will be conducted by specially trained medical officers throughout the study.

\subsection{Outcome Measurements}

2.7.1. Primary Outcome. The primary outcome is the change of the total frequency of self-reported angina attacks at the 4 th week compared with week 0 . This outcome will be determined from the participants' diaries.

2.7.2. Secondary Outcomes. The secondary outcomes include the following: (1) intensity of angina pain on a VAS, which will be reported by the patient on a 100-point pain severity scale, where 0 indicates the absence of pain and 100 indicates the worst pain; (2) consumption of nitroglycerin or Suxiao Jiuxin pills (each participant can only receive one of these types to relieve acute symptoms); (3) the CCS angina class; (4) the SAQ score; (5) the SAS score and the SDS score; and (6) the change in the ST segment of the ECG.

The efficacy outcomes will be measured at weeks 0,4 , and 8. At baseline, the participants will undergo a physical examinations, outcome assessments, and regular tests. At 4th week and 8th week, the outcome assessments will be repeated. The regular tests include echocardiography; blood, urine, and stool tests; blood glucose and lipid tests; and liver and kidney function tests (Table 2).

2.7.3. Assessment of Adverse Events. The risk of side effects of the AP therapy has been found to be low [10-12]. However, we will still examine any side effects of the treatment during the trial. All treatment-emergent AEs will be recorded during the treatment and the follow-up phase. Treatmentemergent AEs include itching, allergies, local infections, and other symptoms. AEs caused by antianginal drugs (basic treatment) will also be documented, and they include headaches, dizziness, nausea, flushing, and abdominal pain [4]. A serious adverse event (SAE) is defined as any $\mathrm{AE}$ that causes incapacity/disability, is life threatening or fatal, or requires hospitalization. Any AE/SAE will be documented in detail and reported. SAEs will be reported to the principal investigator and the ethics committee immediately. The principal investigator will decide whether the participant should withdraw from the study. The number of AEs will be calculated for each group.

2.8. Sample Size Calculation and Statistical Analysis. A previous study [24] found that the average weekly frequency of angina episodes of patients with CSAP was approximately 6.5 before treatment and the average reduction of weekly episodes was 3.7 in the AP group and 1.5 in the routine basic treatment group. Therefore, we predict that the weekly frequency of angina episodes of the HAA, PAA, HAN, and PAN groups will be $2,4.5,4.5$, and 5 after treatment, respectively, with a combined standard deviation of 4 . Assuming a 2-sided 0.05 significance level, a total of 172 patients ( 43 patients per arm) are needed to achieve a $90 \%$ probability of detecting improvement ( $G^{*}$ Power software, Ver. 3.1.7). When considering a potential dropout risk of $15 \%$, a total of 200 patients will be included in the study, with 50 patients in each group.

Statistical analyses will be performed by a statistician who is blinded to the treatments. Statistical nalysis Software 
TABLE 2: Study Schedule.

\begin{tabular}{|c|c|c|c|c|}
\hline & Screening & Baseline (randomization) & Treatment & Follow up \\
\hline Week & -4 & 0 & 4 & 8 \\
\hline Informed consent & $x$ & & & \\
\hline In/exclusion criteria & & $x$ & & \\
\hline Vital signs & $x$ & $x$ & $x$ & \\
\hline Medical history & $x$ & & & \\
\hline Concomitant disease/medication & $x$ & $x$ & $x$ & $x$ \\
\hline Diary card distributed/review & $x$ & $x$ & $x$ & $x$ \\
\hline Total frequency of angina attack & & $x$ & $x$ & $x$ \\
\hline VAS scoring & & $x$ & $x$ & $x$ \\
\hline Consumption of nitroglycerin or Suxiao Jiuxin dropping pills & & $x$ & $x$ & $x$ \\
\hline CCS angina class & & $x$ & $\times$ & $\times$ \\
\hline SAQ scoring & & $x$ & $x$ & $\times$ \\
\hline SAS and SDS scoring & & $x$ & $\times$ & $\times$ \\
\hline ECG & & $x$ & $\times$ & $x$ \\
\hline Echocardiography & & $x$ & & \\
\hline Blood, urine, and stool tests & & $x$ & & \\
\hline Blood glucose and lipid tests & & $\times$ & & \\
\hline Liver and renal function tests & & $x$ & & \\
\hline AEs & & $\times$ & $\times$ & $\times$ \\
\hline Reasons to withdrawal & & & $x$ & $\times$ \\
\hline Safety evaluation & & $x$ & $x$ & \\
\hline Compliance & & $x$ & $x$ & $x$ \\
\hline
\end{tabular}

CCS = the Canadian Cardiovascular Society; SAQ = Seattle Angina Questionnaire; SAS = self-rating anxiety scale; SDS = self-rating depression scale; VAS = visual analogue scale; ECG = electrocardiograph; and AEs = adverse events.

(SAS, Ver. 9.1) will be used. The full analysis set (FAS) population is defined as all randomized patients who receive at least 1 treatment of the study regimen. The per-protocol (PP) population is defined as all patients in the intention-totreat (ITT) population who are not major protocol violators. For the primary analysis, the PP population will be used. For the secondary analysis, the FAS and PP populations will be used. Demographic data at week 0 will be compared to measure the comparability among groups. For continuous variables, standard summary statistics will include mean, standard deviation, median, and maximum and minimum, and analysis of variance (ANOVA) with factor treatment will be performed. For categorical variables, the number and percentage will be tabulated, and chi-square tests will be used. The FAS population analysis will be based on the last observation carried forward (LOCF) for missing data.

\section{Discussion}

AP originates from long-term clinical practice by ancient Chinese doctors, who combined herbal medicine with acupoint stimulation on the basis of TCM theory. Through repeated summarization and innovation, a novel, external TCM therapy has been established [25]. The earlier studies for AP in the treatment of CSAP used self-controlled designs [10, 12, 26, 27], an active control compared with conventional drug therapy $[24,28,29]$, a comparison of acupuncture with conventional drugs [30], a comparison of a transdermal therapeutic system of nitroglycerin with Fufang Danshen Dripping Pills [31], and a comparison of basic AP treatment with a nonacupoint regimen [11]. In these studies, the AP treatment groups were significantly superior to the control groups. However, biases resulted from poorquality trial design, including a lack of baseline data, no placebo control groups, confused randomization methods, and conflicting concomitant medications. Therefore, we have designed a double-blind, randomized, placebo-controlled study to investigate the true efficacy of AP in the treatment of CSAP.

Is AP combined with acupoint stimulation and herbal medicine therapy superior to a single therapy, or is there a placebo effect? The answer cannot be determined from previous studies. We will observe clinical efficacy differences among different treatment regimens, including a combination of herbal medicine and acupoints (HAA group), acupoint stimulation only (PAA group), herbal medicine therapy only (HAN group), and a placebo treatment (PAN group).

In this trial, Suxiao Jiuxin pill is a compound developed by traditional Chinese medicine for cardiocerebral vascular diseases, which is listed in the national essential drug list of China [32]. The components of Suxiao jiuxin pill include Radix chuanxiong, and Borneolum syntheticum plus others [33]. It has been shown to relief angina pectoris and reduce the medication dosage and frequency of nitroglycerin [34, 35]. It was widely used to treat angiocardiopathy disease in 
China. With fewer side effects and the capability of rapid relief angina symptoms, it is accepted by many patients who suffer from angina pectoris and taken for prevention and treatment of angina pectoris. Suxiao Jiuxin pill is $40 \mathrm{mg}$ per pill; a sublingual dose of 5-10 pills (200-400 mg) is recommended during attack of angina pectoris. In this study, we have chosen nonacupoint therapy as the control regimen, as investigators may not be blinded to the treatment, but they will remain blinded to the plasters. The participants will also be blinded to the treatments (acupoint selection and plasters) throughout the study.

\section{Conclusion}

This paper presents a design and protocol of AP for patients with CSAP. The study will evaluate the efficacy and safety of AP for this condition. The results of this trial will provide high-quality evidence for the use of AP in the treatment of CSAP.

\section{Conflict of Interests}

The authors declare that no competing financial interests exit.

\section{Authors' Contribution}

Yulan Ren and Dehua Li contributed equally to this paper. Yulan Ren, Dehua Li, Hui Zhen, Junyan Leng, Linglin Zhang, Jie Zhang, Junling Lv, Hailong Fan, and Fanrong Liang participated in designing the trial, writing the data analysis plan, and drafting the paper. All authors read and approved the final paper.

\section{Acknowledgments}

This study is supported financially by the National Basic Research Program of China "973 Program" (no. 2012CB518501) and the research program of the Science and Technology Department of the Sichuan Province (no. 2011SZ0302) and Chengdu City (no. 12DXYB215JH002) in China. The funders will not have any impact on the trial and the publication of the results. The authors acknowledge the assistance and contributions from acupuncturists, experts, and investigators in each center. They also acknowledge the contributions from patients with CSAP who will participate in this trial.

\section{References}

[1] A. D. Lopez, C. D. Mathers, M. Ezzati, D. T. Jamison, and C. J. Murray, "Global and regional burden of disease and risk factors, 2001: systematic analysis of population health data," The Lancet, vol. 367, no. 9524, pp. 1747-1757, 2006.

[2] C. A. Daly, B. de Stavola, and K. M. Fox, "Predicting prognosis in stable angina-results from the Euro heart survey of stable angina: prospective observational study," The British Medical Journal, vol. 332, no. 7536, pp. 262-267, 2006.

[3] K. Fox, M. A. Alonso Garcia, D. Ardissino et al., "Guidelines on the management of stable angina pectoris: Executive summary: The Task Force on the Management of Stable Angina Pectoris of the European Society of Cardiology," European Heart Journal, vol. 27, no. 11, pp. 1341-1381, 2006.

[4] J. M. Tarkin and J. C. Kaski, "Pharmacological treatment of chronic stable angina pectoris," Clinical Medicine, vol. 13, no. 1, pp. 63-70, 2013.

[5] T. D. Fraker and S. D. Fihn, "2007 chronic angina focused update of the ACC/AHA 2002 guidelines for the management of patients with chronic stable angina: a report of the American College of Cardiology/American Heart Association Task Force on Practice Guidelines Writing Group to develop the focused update of the 2002 guidelines for the management of patients with chronic stable angina," Journal of the American College of Cardiology, vol. 50, no. 23, pp. 2264-2274, 2007.

[6] T. Münzel and T. Gori, "Nitrate therapy and nitrate tolerance in patients with coronary artery disease," Current Opinion in Pharmacology, vol. 13, no. 2, pp. 251-259, 2013.

[7] K. Prasad, V. Sharma, K. Lackore, S. M. Jenkins, A. Prasad, and A. Sood, "Use of complementary therapies in cardiovascular disease," American Journal of Cardiology, vol. 111, no. 3, pp. 339345, 2013.

[8] F. Y. Chu, X. Yan, Z. Zhang et al., "Features of complementary and alternative medicine use by patients with coronary artery disease in Beijing: a cross-sectional study," BMC Complementary and Alternative Medicine, vol. 13, no. 1, article 287, 2013.

[9] M. R. Prausnitz and R. Langer, "Transdermal drug delivery," Nature Biotechnology, vol. 26, no. 11, pp. 1261-1268, 2008.

[10] Z. C. Fan, M. Y. Li, Z. L. Xi et al., "Observation on therapeutic effects of angina pectoris treated with acupoint-application," Chinese Acupuncture \& Moxibustion, no. 9, pp. 527-577, 1997.

[11] J. M. Yin, L. Y. Luo, D.C. Zhang et al., "Clinical study on acupoint application by tongxintie plaster on patients with angina pectoris," Chinese Journal of Integrative Medicine on Cardio/Cerebrovascular Disease, vol. 7, no. 10, pp. 1138-1140, 2009.

[12] Y. Liu, T. Wang, and J. Zhang, "Treatment of angina pectoris with medicinal plaster fixed at acupoints-a report of 54 cases," Journal of Traditional Chinese Medicine, vol. 18, no. 1, pp. 12-14, 1998.

[13] J. L. Liu, M. F. Luo, Z. Y. Wang et al., "Effects of acupointapplication on cardiac performance, myocardial metabolism and structural changes in myocardial ischemia rabbits," Chinese Journal of Natural Medicine, no. 3, pp. 41-45, 2005.

[14] China Society of Cardiology, "Chronic stable angina pectoris diagnosis and treatment guidelines," Chinese Journal of Cardiology, pp. 195-206, 2007.

[15] C. X. Li, F. Ding, L. Wang et al., "Research of protection of new GuanxinSuhe dripping pill on experiment myocardial ischemia," Liaoning Journal of Traditional Chinese Medicine, vol. 39, no. 5, pp. 771-773, 2012.

[16] C. Xu, D. Q. Suo, and Y. L. Cao, "The effects of Guanxinsuhe soft capsules on acute myocardial ischemia in dogs," Journal of Shenyang Pharmaceutical University, vol. 18, no. 5, pp. 373-375, 2001.

[17] J. Y. Zhang, Y. K. Li, K. Ma et al., "Effects of series of Muskone on cardiomyocytes suffering from hypoxia- reoxygenation injury," Chinese Journal of New Drug, vol. 16, pp. 1481-1484, 2007.

[18] X. Zhang, J. Q. Song, Y. Wang et al., "Mechanism of GuanxinSuhe-containing serum against apoptosis of cardiomyocytes induced by hydrogen peroxide," Chinese Traditional Patent Medicine, vol. 35, no. 2, pp. 232-236, 2013. 
[19] J. R. Zhang, "Treatment of angina pectoris guanxin suhexiang capsules-a report of 40 cases," Modern Journal of Integrated Traditional Chinese and Western Medicine, vol. 12, no. 17, 1859, 2003.

[20] D. F. He, Y. L. Ren, Y. Tang et al., "Application of data-mining to analyze characteristics of meridians and acupoints selected for acu-moxibustion treatment of chest blockage in ancient literatures of different periods of ancient days," Liaoning Journal of Traditional Chinese Medicine, vol. 40, no. 6, pp. 1209-1213, 2013.

[21] S. Lim, "WHO standard acupuncture point locations," Evidence-Based Complementary and Alternative Medicine, vol. 7, no. 2, pp. 167-168, 2010.

[22] Y. Lin, F. Ji, G. R. Huang, and P. Li, "Our considerations about non-acupoint selection for experimental studies in rats," Zhen Ci Yan Jiu, vol. 38, no. 4, pp. 334-338, 2013 (Chinese).

[23] X. Duan, L. Zhou, T. Wu et al., "Chinese herbal medicine suxiao jiuxin wan for angina pectoris," Cochrane Database of Systematic Reviews, vol. 23, no. 1, Article ID CD004473, 2008.

[24] X. G. Fu, "Acupoint application in treating angina pectoris combined with Yiqihuoxue decoction-a report of 42 cases," Guiding Journal of Traditional Chinese Medicine and Pharmacology, vol. 18, no. 10, pp. 67-68, 2012.

[25] Y. H. Wu and B. Dong, "Discussion on prevention and treatment of acupoint application in treating angina pectoris," Liaoning Journal of Traditional Chinese Medicine, vol. 40, no. 9, pp. 1891-1892, 2013.

[26] C. Y. Yu and B. Yu, "Acupoint application with herbal medicine in treating coronary heart disease-a report of 420 cases," Chinese Acupuncture \& Moxibustion, no. 5, pp. 5-6, 1990.

[27] D. Y. Li and C. X. Gao, "The effect of Guanxi plaster on blood viscosity in patients with angina pectoris," LiShiZhen Medicine and Materia Medica Research, vol. 17, no. 11, p. 2272, 2006.

[28] J. J. Liu, "Clinical observation of unstable angina pectoris with coronary heart disease (Qi Deficiency blood stagnation syndrome) by using Angina pectoris pastes," Liaoning Journal of Traditional Chinese Medicine, vol. 38, no. 4, pp. 662-663, 2011.

[29] A. P. Jiang, R. Y. Xie, X. Li et al., "Clinical study on acupoint application with herbal medicine for angina pectoris," Chinese Journal of Information on TCM, vol. 10, no. 1, pp. 41-42, 2003.

[30] H. G. Jin, F. Qi, and M. Zhao, "Clinical study on acupoint application in treating stable angina pectoris compared with acupuncture," Journal of Changchun University of Traditional Chinese Medicine, vol. 29, pp. 523-524, 2013.

[31] J. Y. Dai, Z. F. Wang, and D. Li, "Clinical study of acupoint application with Danqiyixin plaster in treating angina pectoris," Chinese Acupuncture \& Moxibustion, no. 10, pp. 588-590, 1997.

[32] L. Feng, T. Han, and Y. L. Zhou, "The conclusion of the clinic effect for Suxiao Jiuxin Wan for CHD and angina pectoris," Journal of Emergency Syndromes in Chinese Medicine, vol. 9, no. 1, pp. 4-6, 2000.

[33] Z. Q. Zhuang, Y. Wan, Q. Zhang, G. Y. Yao, and G. Q. Feng, "Curative effect observing of Suxiao jiuxin wan for no symptom myocardial infractiono of coronary heart disease 48 cases," Modern Journal of Integrated Chinese Traditional and Western Medicine, vol. 8, no. 11, pp. 1781-1782, 1999.

[34] G. Yuan, "Curative effect observions on Suxiao jiuxin wan compared to Xiao xin tong for Angina pectoris," Medical Journal of Healing of Heart and Vessel, vol. 9, no. 9, pp. 71-72, 2002.

[35] J. Wei and Y. Tang, "Comparing research on the clinical effect of Suxiao Jiuxin Wan for ischemic heart disease," Journal of
Emergency Syndromes in Chinese Medicine, vol. 164, no. 4, pp. 159-160, 1995. 


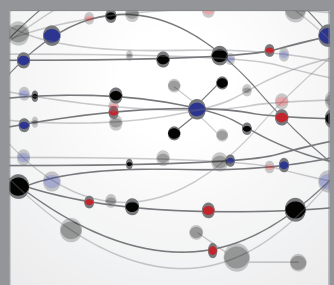

The Scientific World Journal
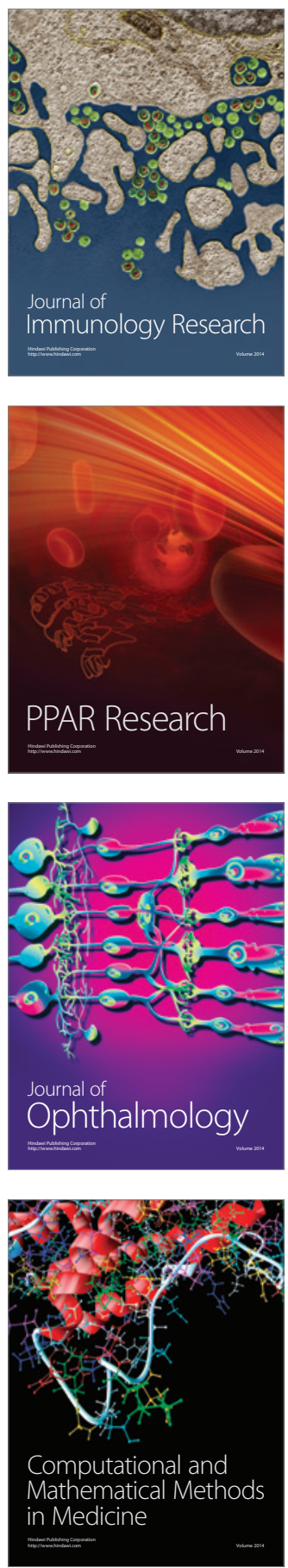

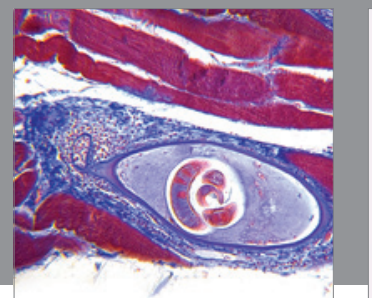

Gastroenterology

Research and Practice
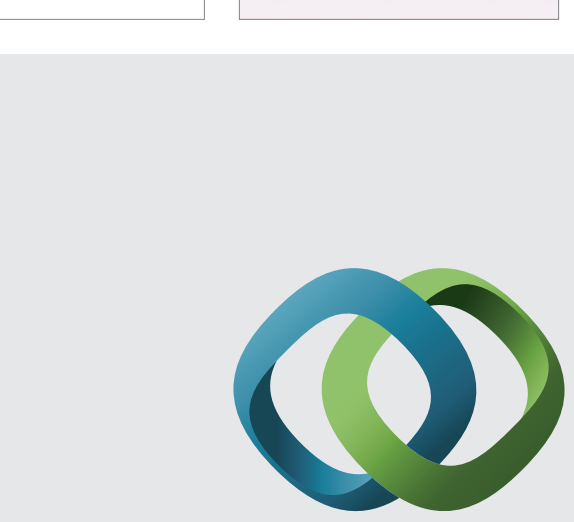

\section{Hindawi}

Submit your manuscripts at

http://www.hindawi.com
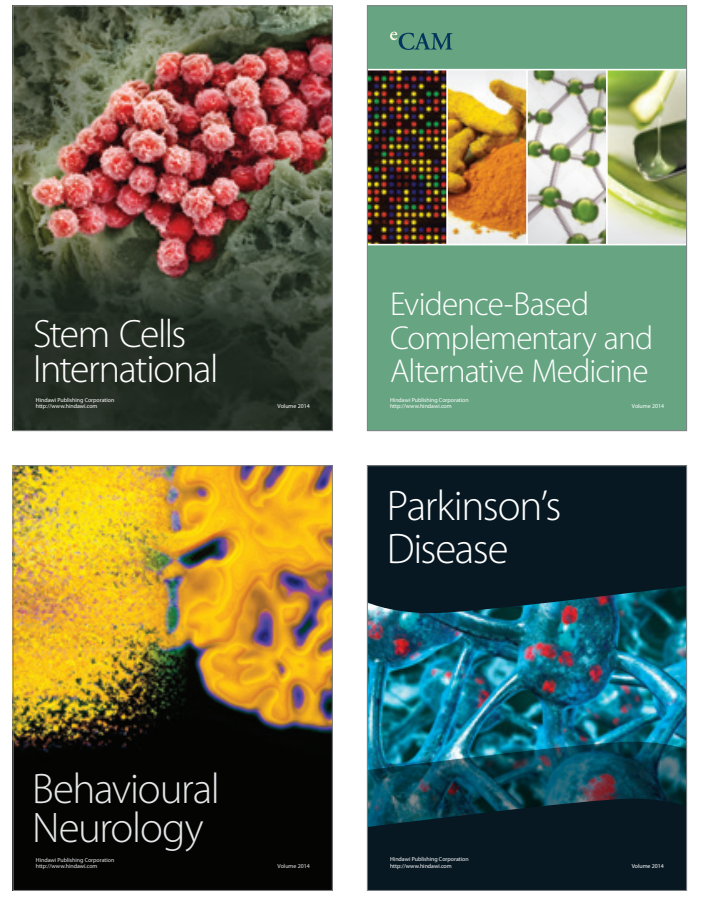
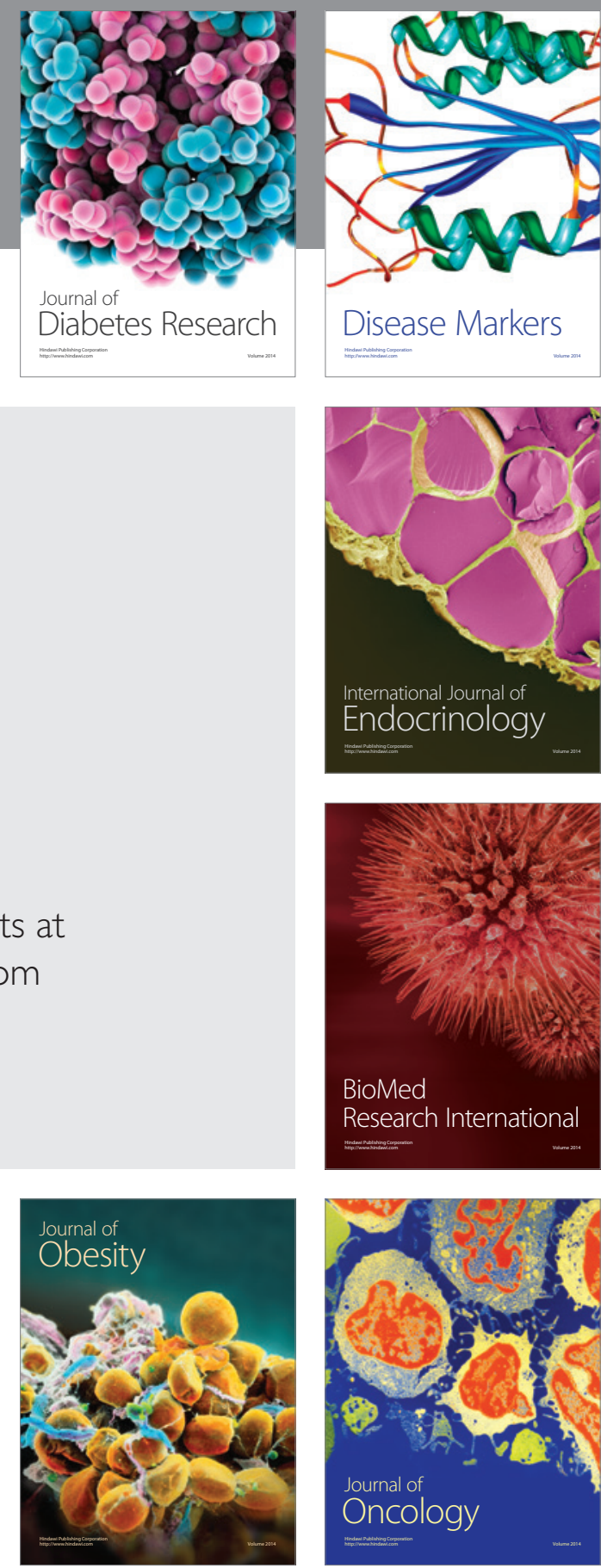

Disease Markers
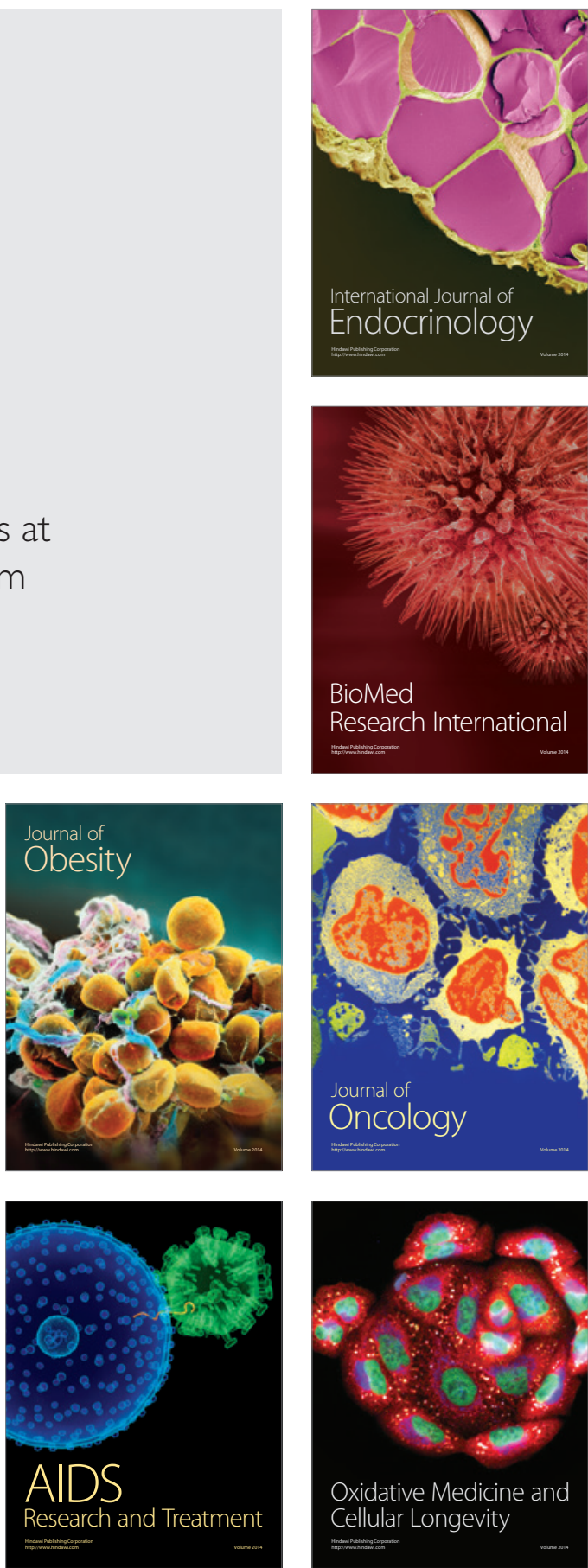